\title{
Enterprise E-Commerce Marketing System Based on Big Data Methods of Maintaining Social Relations in the Process of E-Commerce Environmental Commodity
}

Guihe He, Hunan University of Humanities and Technology, Hunan, China

\begin{abstract}
Since entering the 21 st century, with the rapid development of internet technology, the network platform of e-commerce has undergone great changes. With the rapid development of e-commerce economy, the widespread existence of credit risks in the trading process of e-commerce environmental goods has caused great harm to the healthy development of e-commerce and has gradually become the biggest bottleneck restricting the development of e-commerce in China. Based on the basic model of e-commerce market, this paper proposes four credit mechanisms to maintain social relations in the process of e-commerce environmental goods trading, that is, credit mechanism without government intervention. As can be seen from the experimental data, the alpha coefficient of each potential variable set in the experiment in this paper is above 0.6 , which is within the acceptable range. This verifies that the method model proposed in this paper to maintain social relations in the process of e-commerce environmental goods trading is effective in application.
\end{abstract}

\section{KEYWORDS}

Commodity Trading, E-Commerce Environment, Electronic Commerce, Social Relations

\section{INTRODUCTION}

E-commerce refers to commercial and trade activities using modern electronic information technology, especially network technology (kabanda \& brown, 2017). Since the beginning of the 21 st century, with the rapid development of internet technology, the e-commerce network platform has undergone tremendous changes. Especially with the rapid decline of network costs, and the acceleration of network infrastructure construction, the development speed of e-commerce has significantly been improved (chen et al., 2015; uzoka et al., 2017). The development of e-commerce is the driving force for the world economy's future development and a vital driving force for transforming the world economy into a knowledge-based economy. E-commerce soon becomes the main form of business activity (hua, 2016; wei \& ho, 2019). However, with the rapid development of the e-commerce economy, e-commerce fraud appears to be out of place in the e-commerce symphony, seriously damaging the e-commerce activities (kj et al., 2015). As people expect credit risk to become higher and higher, more and more internet users have abandoned online transactions (zhang et al., 2019). As the domestic e-commerce market is still in the development stage, the environment in all aspects is still immature, and online fraud is more serious (maier, 2018). Internet users have experienced 
consumer fraud on the internet, and the number of internet users has reached 38.8 million (chen et al., 2016). China internet network information center warned that many phishing websites use fake official website addresses and web contents to defraud online banking or credit card passwords. In the case of uncertain website authenticity, most internet users will abandon online transactions, and the development of e-commerce requires a more credible and reliable network environment (tamini \& sorgho, 2018; taylor, 2016).

With the rapid development of Internet technology, e-commerce companies continue to develop, which changes corporate marketing models. In the era of the global e-commerce economy, an enterprise's destiny and the supporting point of its development depend on users' decisions and between users and enterprises, according to the network and its regulations, through different business operations and mutual information channels. In today's society, the application of e-commerce in enterprises has gradually changed from basic theoretical research to practical development. Many companies have obtained the recognition of the capital market and successfully gone public. Various e-commerce platforms are also operating in an excellent operating mode. Traditional business structure and trading methods have changed due to e-commerce, and corporate marketing models have also changed. The research on the e-commerce marketing model between enterprises is increasing, which has promoted more and more mature e-commerce marketing models, with significant development and innovation. Corporate marketing and e-commerce are inseparable, and corporate marketing focuses on communication and feedback with customers. Corporate marketing technology and previous marketing models have been affected by the research of new marketing models, and the development of corporate marketing innovation practices has been significantly improved.

Yunita believed that customer loyalty is required in e-commerce, and using social media is one of the new ways for maintenance. He studied the correlation to find out how social media affected its members' loyalty and surveyed 257 Facebook-registered users with multiple linear regression methods. Social media plays a massive role in increasing the likelihood of repeated purchases and loyalty behavior, but this strategy does not affect mouth-to-mouth sales(Kaluzhsky, 2015; Kim, 2019).

Zhang believed that in the e-commerce environment, the seller's reputation is vital for potential buyers to make decisions. Most existing trust evaluation models only calculate a value to reflect the seller's overall trust level, regardless of any trading context. In such a simple trust evaluation model, buyers may be vulnerable to malicious, fraudulent sellers in an unbalanced transaction value imbalance; that is, malicious sellers can accumulate a high level of trust by selling cheap products and deceiving buyers and inducing them to buy expensive products. He first proposed a trust vector consisting of four trust values (called CTT values), calculated based on past transactions and upcoming transactions. Also, while calculating the CTT value, the buyer can specify and adjust parameters such as product category, price range, and time range to generate different sets of trust values that summarize the seller's reputation. Therefore, the value imbalance problem in the upcoming transaction can be determined. The calculation of CTT values requires the pre-calculation of the aggregation of large-scale ratings and transaction data, combined with the necessary three dimensions (i.e., product category, price, and time) to respond to buyer queries promptly for different CTT values. Three new data structures and algorithms were proposed to solve this challenge, focusing on efficient CTT value calculation. His experiments on eBay datasets and large synthetic datasets illustrate his proposed approach's advantages and disadvantages in responding to buyers' CTT queries (Mohanty \& Gupta, 2015; Yunita \& Gunawan, 2018).

Mohbey believed that mobile e-commerce plays an essential role in our lives because it helps us use new trends and technologies to improve business and provide faster information. In the mobile e-commerce environment, user-behavior sequence mining is an emerging issue involving mobile user services, accessing sequences at different times and locations. In the current scenario, the user may only be interested in service sequences with certain specific constraints. A constraint can be defined as the type of service or the importance of a particular service. Here, he considered services important or useful to a particular user in a mobile e-commerce environment. An effective framework, 
CIUBSM (Constraint-based Interesting User Behaviour Sequence Mining), was proposed to deal with significant services constraints and generate interesting sequences of behaviors for mobile users (Song et al., 2015; Zhang et al., 2015).

Aanen believed that in the past few years, our e-commerce had grown steadily, and this growth has created many problems for services to aggregate product information and products. One of the problems facing the aggregation service is the matching of product categories from different web stores. An algorithm automating this task is proposed, which collects product information from multiple websites to search, compare, or recommend system applications. His algorithm uses word sense disambiguation techniques to handle different names between different taxonomies. The proposed solution focuses on improving the disambiguation process compared to existing state-ofthe-art methods and addressing product-specific features, such as combinatorial categories. In this context, lexical similarity and similarity aggregation are rechecked (Mohbey \& Thakur, 2015; Zhou et al., 2017).

The work's innovations are as follows:

(1) In-depth and systematic research on credit issues in e-commerce environmental commodity trading from the institutional level. In the research of e-commerce related fields, its credit problem receives much attention. The work summarized the characteristics of different e-commerce credits and credit operation mechanisms from the system level and the credit mechanism with guiding significance for e-commerce credit development.

(2) Comprehensive market structure theory and information economics theory, constructing a credit mechanism model in the e-commerce environment. It is based on the analysis of the various components of the e-commerce market, namely, the number of buyers, consumers, information, network externalities, entry costs, product attributes, technology, and other factors on the credit mechanism in the e-commerce environment, credit intermediation, and credit regulation. The interaction between them is organically integrated to construct an overall e-commerce credit mechanism model, which makes up the shortcomings of existing research ignoring the two's mutual influence.

(3) Using the game analysis method and the maximization of net social income (social welfare) as the yardstick, the four credit mechanisms under the e-commerce environment were analyzed by mathematical reasoning. For these four credit mechanisms of e-commerce, the work has established a dynamic Boben model, which made mathematical derivation, analysis, and comparison of the benefits (net income) and net social income (Social Welfare) of business enterprises and consumers under various mechanisms. However, there are many studies to analyze different credit mechanisms from a normative perspective, and there is no clear standard for the merits and demerits of the credit mechanism.

\section{PROPOSED METHOD}

\subsection{E-Commerce}

(1) The English names of e-commerce are e-commerce (EC) and e-business(EB). EC refers to the business relationship between customers and service providers, and merchants (including stores and shopping centers). For example, a customer purchases an electronic store item on the network, and the user enjoys a service provided by a service provider on the network. EB refers to a broader range of business relationships, including internal business relationships, supply chains, customer relationships, online transaction services, and other business activities, covering business relationships with customers and service providers, including service providers. 
Business relationships between merchants and manufacturers, mong producers and raw materials businesses, merchants, manufacturers, and raw material producers, and between government departments and other business activities exist in the real world. The work shows business relationships between different entities.

After receiving the user's order, the enterprise assembles and produces in time according to the user's different needs. This model shows great flexibility in production. The essence of real-time production can accurately deliver the necessary parts to the necessary places at a critical moment. This method replaces large-scale production lines with small-batch production, allowing enterprises to make rapid adjustments according to market needs. From a management perspective, real-time production transforms people from "living tools" to machines' master and soul, enabling the management of the knowledge economy to be carried out in the enterprise.

E-commerce realizes and simplifies customized digital production. By building various databases, enterprises can record all customers' data, communicate with customers in real-time through the Internet, and grasp the customers' latest demand trends. After the enterprise obtains user demand information, it can send the information accurately and quickly. As far as the design, supply, production, distribution, and other enterprise links are concerned, all links can respond to information quickly, accurately, and orderly. They respond quickly and sensitively to market demand, change the general market orientation to the market orientation of customer participation, and use computer process simulation technology and concurrent engineering organization forms to achieve any combination of products, services, and information, thereby greatly enriching varieties. It can significantly shorten the product design, quickly provide products and services rich in variety, arbitrary batch size, high performance, and high quality, and meet customer satisfaction.

\section{(2) Connotation and extension of e-commerce}

Connotation and extension are logical pairs of terms. Connotation refers to the specific attributes in the concept, which refers to the concept's content. Representation is the object referred to by the concept, that is, the scope of the concept.

The size of the company does not limit Internet marketing. At a lower cost, companies can participate in the global information network and trade network to find and contact potential customers and deliver products to the market that only large companies with vital financial resources can own. In other words, network marketing has changed the previous situation of small and medium-sized enterprises in disseminating and obtaining market information to a certain extent and has expanded the marketing market of small and medium-sized enterprises.

The definition of e-commerce shows e-commerce covers the preconditions of e-commerce, the personnel engaged in e-commerce and their knowledge and skills, the electronic means of the network means and systems, and business objects. The prerequisite for e-commerce is information technology, especially the application of network technology represented by Internet technology. The core of e-commerce is to master modern information technology and business theory and the practice of compound talents, which are the key elements of e-commerce. The basis of e-commerce is the comprehensive utilization of the network environment and the electronic tools of various systems; e-commerce engages in various commercial activities centered on commodity trade; e-commerce is efficient, low-cost, safe, and convenient. Products and services are provided to enhance the competitiveness of enterprises.

The extension of e-commerce can be derived from the definition and connotation of e-commerce. The expansion of e-commerce mainly focuses on developing network environment and electronic tools, product categories, and business activities. The following aspects are included: the development of electronic tools, the revolution in the development of computers and network technologies, the integration of mechatronics, optoelectronics and electronic products, and the widespread use of network 
technologies represented by Internet technologies. The expansion of product categories reflects the introduction of generic product concepts and product diversity. Features, such as search engines, short message services, introduce the concept of service goods; the expansion of business activities leads to expanding business activities, forming a multi-network connection from the government, market, production, and consumers.

The characteristics of e-commerce by creating a virtual market environment e-commerce eliminate both time and space barriers, thereby increasing trade opportunities, reducing transaction costs, improving service quality, and improving business activities' efficiency. Compared with traditional commerce, e-commerce has the following salient features: 1) The commercial portal reduces the threshold for enterprises to enter international trade and creates favorable conditions for enterprises to establish a competitive advantage development strategy. There are no restrictions on companies displaying their brands and images. 2) The two-way interaction and dissemination function of network multimedia information makes it more convenient for enterprises to promote products and services. It is of great significance for enterprises to expand their visibility and competitiveness in the global business market space. 3) E-commerce has greatly improved business activities, enabling enterprises to enjoy faster and more convenient information transmission, information exchange, information sharing, and information processing capabilities than traditional trade methods. 4) E-commerce has created conditions for paperless trade. The Internet-based OPEN EDI transaction method provides a new way for enterprise development. Both parties can complete the transaction business by e-mail, IP phone, and network fax communication. If the legal documents related to the agreement can be exchanged using the web-based open electronic data exchange (open.EDI). 5)Change e-commerce and promote the reengineering of business processes. By integrating the value chain relationships of upstream and downstream companies, business relationships between companies in the supply chain and the sales chain are more efficient and economical. 6) The business portal is the enterprise's commodity trading platform and the customer-relationship management-service system. It provides customized services for customers' personalized consumer needs and provides product information, service information, user troubleshooting, and other services efficiently and conveniently. 7) Add value to network services by integrating enterprise relationships into the value chain. It integrates supply chain management, supplier management, sales chain management, customer relationship management, and e-commerce transaction market management into an enterprise information service platform that integrates information and shares information. By providing information sharing, communication, feedback, and decision-making, we can improve enterprises' information processing capabilities and quality to adapt to the highly competitive market environment. 8) E-commerce is not limited by time, space, and region, providing customers with all-around, all-weather quality services.

\subsection{E-Commerce Model}

\section{(1) Environmental model of e-commerce}

Any enterprise has inextricably linked with the market. As a market unit, the enterprise must maintain an input and output relationship with the market system and exchange materials, labor, and information. The market is the starting point of business activities and the medium for cooperation and competition between enterprises and the outside world. These activities involve two different parts of the market system: the consumer market and the industrial market. Figure 1 shows the environmental model of e-commerce. It reflects the enterprise-centered application model in the consumer market and the industrial market and is an essential theoretical basis for realizing the e-commerce system. 


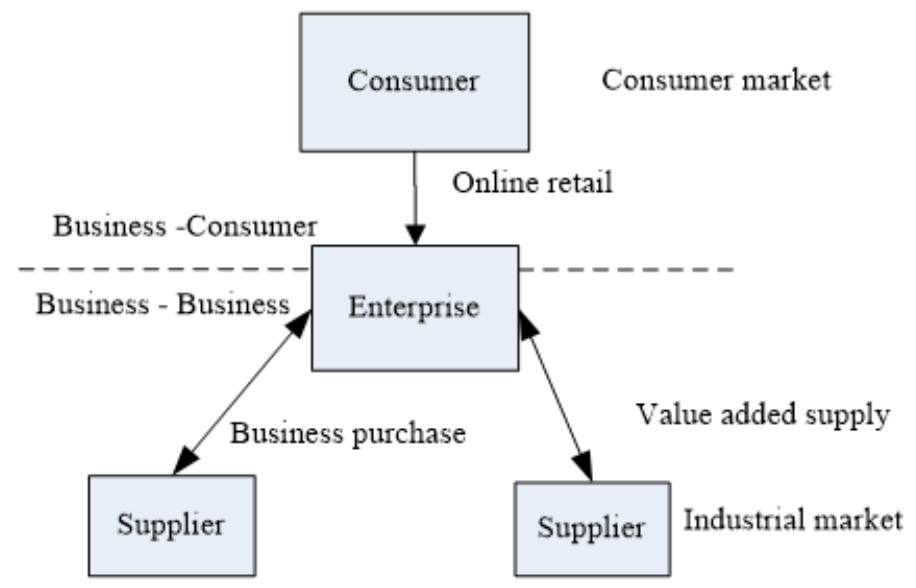

(2) Conceptual model of e-commerce

The conceptual model of e-commerce is a general abstract description of e-commerce activities in the real world. It consists of trading themes, electronic markets (EM), trading transactions, information flows, capital flows, material flows, and other basic elements.

In the conceptual model of e-commerce, transaction soil refers to objective objects that can participate in e-commerce activities, such as enterprises, banks, stores, government agencies. The electronic market refers to the exchange of local goods and services with EC entities' participation, connected by various business activity participants and various communication devices into a unified economic whole through the network. Transaction transactions refer to specific business activities between EC entities, e.g., inquiries, quotations, transfer payments, advertising, and cargo transportation.

Any transaction in e-commerce contains three basic "streams": logistics, capital flow, and information flow. Among them, logistics mainly refers to the distribution and delivery channels of goods and services. For most goods and services, logistics may still be carried out through traditional distribution channels. However, certain goods and services can be delivered directly over the Internet, such as various electronic publications, information consulting services, and valuable information. The flow of funds mainly refers to the transfer of funds, including payment, transfer, exchange, and other processes. The information flow includes the provision of commodity information, promotion, marketing, technical support, after-sales service, commercial trade documents (such as inquiry orders, quotations, payment notices, and transfer notices), the counterparty's ability to pay, and intermediary reputation. Each e-commerce transaction subject faces an electronic market and must choose the transaction's content and object through the electronic market. The conceptual model of e-commerce can be abstractly described as the transaction relationship between the subject of each e-commerce transaction and the electronic market (See Figure 2).

(3) Credit mechanism

In order to ensure the security of data during network transmission, sensitive files are encrypted; even if others intercept the file, the content cannot be obtained; the integrity of the data is guaranteed 


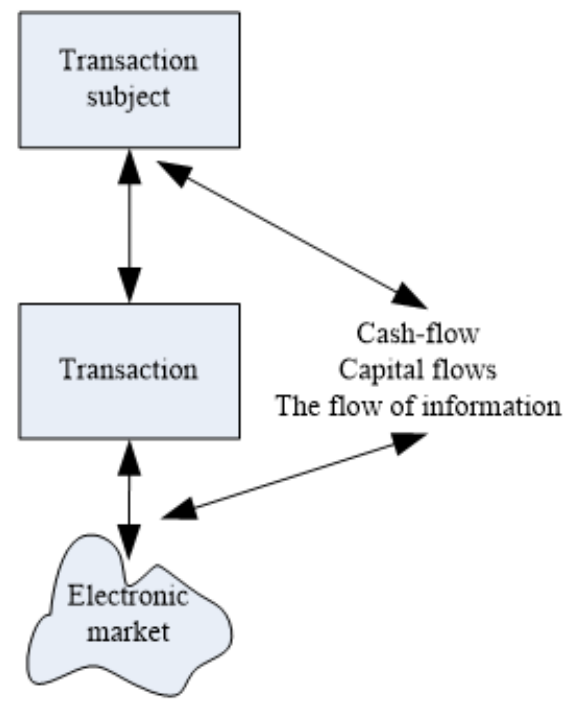

to prevent interceptors from adding other information to the file; the source of the data and information performs verification to ensure the identity of the sender. At present, a combination of a public key encryption system and a secret key encryption system is commonly used internationally to meet data encryption requirements. In the transmission process, digital signature technology and verification technology must guarantee the integrity of the data. Certificates and certificate management agencies are introduced in the actual operation to alleviate the trouble that users need to verify many public keys when exchanging data with different objects.

To engage in e-commerce activities on the Internet, in addition to security measures, a corresponding safe and reliable electronic payment system must be established. Electronic payment methods on the Internet include credit cards, electronic cash, and electronic checks (E-Check), all of which have corresponding security protection methods.

The credit information application is a process by which the credit management department promptly reflects the trustee's credit status and risk evaluation to various internal departments. It combines information flow, capital flow, and credit flow to provide a basis for corporate decisionmaking. The high degree of informationization within the credit grantor can allow the credit management department to search and process credit information and reduce the communication cost in credit information application.

The mechanism is an institutional constraint. The most important thing is to understand two points to understand the concept of mechanism: First, the existence of each part of the thing is the premise of the mechanism's existence. Second, coordinating the relationships between the various parts must be a specific mode of operation; that is, a mechanism is a way of connecting the various parts of a thing to coordinate operations and function. Therefore, the mechanism's essence is to show the relationship and function of various components in a specific organism. Mainstream economics studies credit mechanisms mainly on the premise of information asymmetry. Due to the information asymmetry between the credit provider and the credit recipient, the credit recipient's credit commodity's value is difficult to determine, and its behavior is unpredictable, which leads to inefficiency and credit risk of credit transactions. Therefore, information asymmetry is the premise of studying the credit mechanism. Understanding credit as a mechanism or system is the inevitable result of the market economy's continuous development and the legal system's improvement. It 
does not require pure morality to do with utilitarianism between stakeholders as an economical and reasonable credit mechanism. It recognizes that people are pursuing interests, but such pursuits should be guided by reason and should be fair, reasonable, equal, and mutually beneficial. Therefore, the credit mechanism's goal is the credit behavior of the subject, and constructing the credit mechanism changes the value orientation of the subject's credit behavior.

\section{EXPERIMENTS}

\subsection{Experimental Design}

The work used the questionnaire method to conduct empirical analysis and discuss the relevant research hypothesis of credit intermediary economic function in the e-commerce environment.

In the work, 1,200 questionnaires were sent through the EDM platform, and 1,050 questionnaires were collected, of which 1,020 were valid (samples meeting the EDM empirical research).

\section{(1) Questionnaire design}

To improve the questionnaire method's data reliability, the structure of the questionnaire and the project's design are essential. In the work, six potential variables were designed. The following steps were used to design the measurement items for each potential variable: First, it reviewed the current research results of top foreign journals and found more measurement items for each potential variable. They were commonly used and translated into Chinese. Secondly, to ensure the measurement project, in-depth interviews were conducted with students with a rich e-commerce shopping experience, and the measurement projects were modified accordingly to conform to China's national conditions. The third is to conduct a preliminary investigation of the small sample using a questionnaire consisting of preliminary design measurement items and make simple corrections based on the preliminary investigation problems to form a formal questionnaire.

(a) Test potential variables in project design. The research mainly involves six potential variables, namely credit feedback mechanism, real-name authentication, third-party payment tools, communication and interaction mechanisms, e-commerce platform trust, and the validity of the transaction subject's purchase intention. The work took Taobao, China's largest e-commerce platform, as a typical object of the questionnaire. Based on the previous research results and the mentioned survey project setting process, a project design scheme for measuring each potential variable was proposed.

(b) Design control variables. This research questionnaire designed five control variables, mainly the basic situation of individual consumers and their familiarity with the Internet. Simultaneously, the frequency of purchase (purchase times) and consumption intensity (average purchase amount) of individual consumers using Taobao were studied.

\subsection{Data Collection}

The work explored whether the various credit inter-mediation systems of e-commerce platforms could promote the trust of transaction entities on e-commerce websites and thus affected the consumers' willingness to purchase. Therefore, users who frequently browsed Taobao were selected as research objects. Because of the particularity of the survey object, the work took the form of an online survey. Questionnaires are widely distributed and recycled through the EDM platform of a well-known website to obtain better-performing data. In the work, 5,000 questionnaires were sent through the EDM platform, and a total of 262 questionnaires were collected, of which 231 were valid (samples in the questionnaire that did not visit Taobao were considered invalid). It met the requirements of 
Table 1. Analysis and comparison of operational efficiency of four credit mechanisms

\begin{tabular}{|l|l|l|l|l|l|}
\hline \multicolumn{2}{|c|}{} & \multicolumn{1}{|c|}{$\mathbf{1}$} & \multicolumn{1}{|c|}{$\mathbf{2}$} & \multicolumn{1}{|c|}{$\mathbf{4}$} \\
\hline \multirow{3}{*}{ Credit rule execution } & Cost & High & High & High & Medium \\
\cline { 2 - 6 } & Strength & Low & Low & High & High \\
\hline \multirow{2}{*}{ Cusiness investment } & Protection & High & High & Low & High \\
\hline \multirow{3}{*}{ E-business company } & Credit rights & Low & Low & High & Medium \\
\cline { 2 - 6 } & Confidence & Low & Low & High & High \\
\hline \multirow{2}{*}{ International coordination of credit disputes } & Credit rights & High & High & Low & Medium \\
\hline \multirow{2}{*}{ E-commerce market operation } & Confidence & High & High & Low & High \\
\hline \multirow{2}{*}{ Information } & Transaction cost & High & Medium & High & Medium \\
\cline { 2 - 6 } & Operating cost & High & Low & High & Low \\
\hline Degree of usage & Asymmetry & High & Medium & High & Medium \\
\cline { 2 - 6 } & Disclosure & Zero & High & Low & High \\
\hline
\end{tabular}

Note: 1 indicates an entirely bottom-up credit mechanism; 2 a bottom-up credit mechanism for introducing third-party credit intermediaries; 3 a top-down credit mechanism; 4 a top-down credit mechanism introducing the third-party credit intermediary.

empirical research on the number of samples. Although sending questionnaires through the EDM platform was convenient and huge, the proportion of users who responded via email was generally low.

\section{DISCUSSION}

\subsection{Analysis of Factors Affecting Maintaining Social Relations in E-Commerce Environmental Commodity Trading Process}

(1) Comparative analysis of efficiency among different credit mechanisms in the e-commerce environment

Table 1 shows the operational characteristics and efficiency of each credit mechanism. The credit mechanism introduced by third-party credit intermediaries with government agencies' participation has more advantages in all aspects.

Figure 3 shows in any pure credit mechanism, either the pure top-down credit mechanism or the pure bottom-up credit mechanism can only benefit the party of the game, so neither sides have a higher degree of cooperation. On the other hand, the hybrid e-commerce credit mechanism is more balanced in the distribution of equity and timber, and thus it is easier to achieve a cooperative equilibrium of the game. Under the government's lack of credit mechanism, the e-commerce market structure gives e-commerce companies more rights, while e-commerce companies also have the advantage of bargaining to establish rights. Therefore, e-commerce companies only need to consider the minimum level of credit rights offered to consumers to ensure their participation in e-commerce transactions. However, in the credit mechanism where government agencies participate, third-party credit intermediaries and e-commerce companies reaching private agreements with consumers need to provide consumers with a minimum benefit, and government agencies can guarantee their basic benefits. From the previous analysis results, when government agencies are involved in credit regulation, consumers' benefits are higher than those without the government. 
Figure 3. Comparison of net income between e-commerce companies and consumers under four credit mechanisms

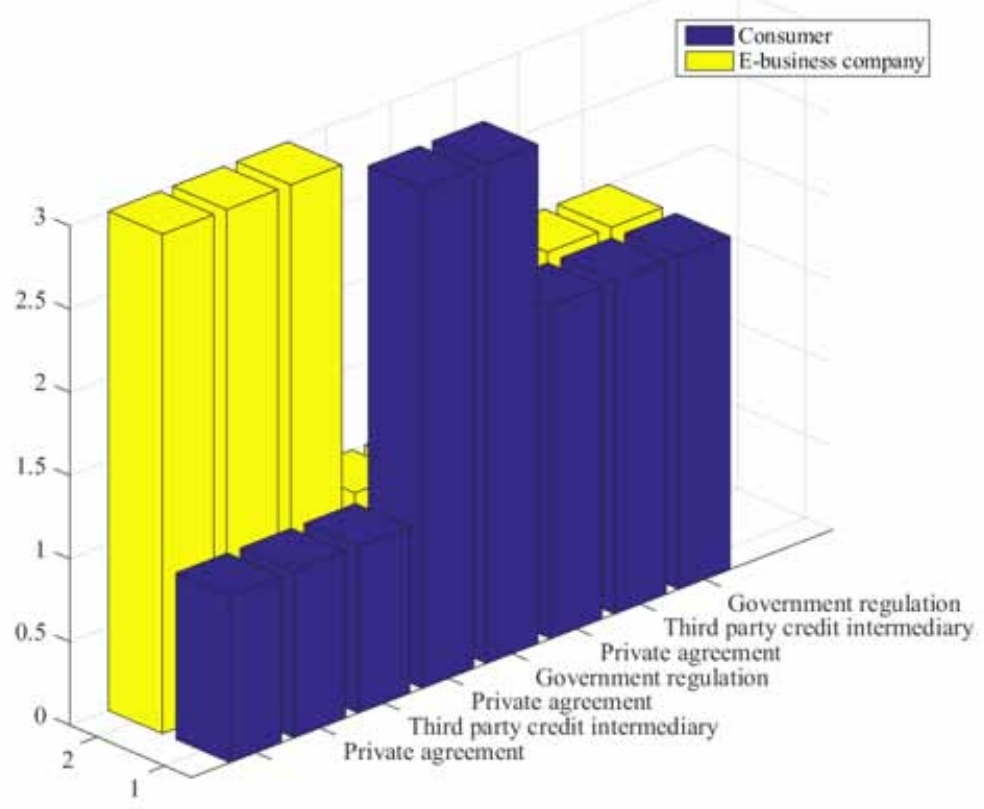

Figure 4. Reliability analysis for each potential variable

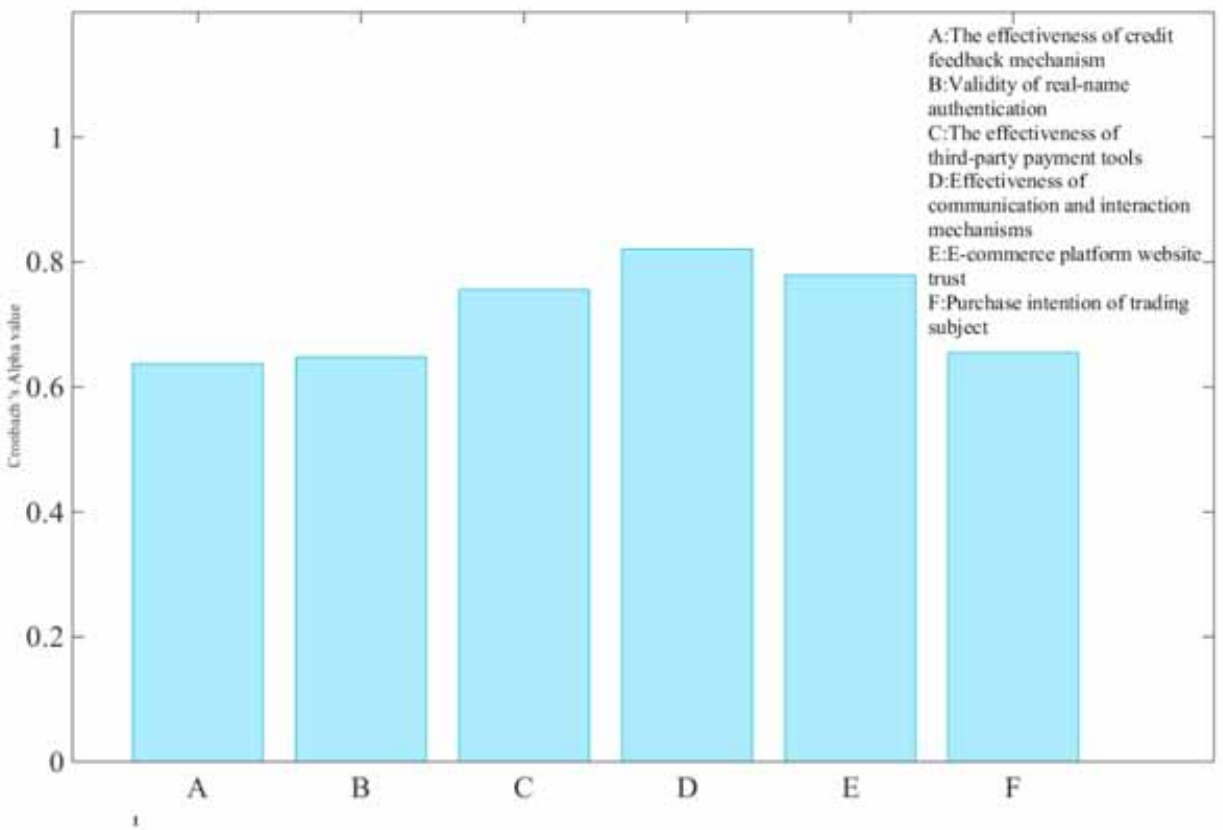


Table 2. Relevance validity analysis of individual items and sums of potential variables

\begin{tabular}{|c|c|c|}
\hline \multirow{4}{*}{ Effectiveness of the credit feedback mechanism } & Measurable variables & $\begin{array}{l}\text { Pearson correlation } \\
\text { coefficient }\end{array}$ \\
\hline & A1 & $.639(* *)$ \\
\hline & A2 & $.651(* *)$ \\
\hline & A3 & $.634(* *)$ \\
\hline \multirow{3}{*}{$\begin{array}{l}\text { Effectiveness of communication and interaction } \\
\text { mechanisms }\end{array}$} & B1 & $.682(* *)$ \\
\hline & $\mathrm{B} 2$ & $.721(* *)$ \\
\hline & B3 & $.739(* *)$ \\
\hline \multirow{4}{*}{ Purchase intention of trading subject } & $\mathrm{C} 1$ & $.698(* *)$ \\
\hline & $\mathrm{C} 2$ & $.654(* *)$ \\
\hline & $\mathrm{C} 3$ & $.702(* *)$ \\
\hline & $\mathrm{C} 4$ & $.673(* *)$ \\
\hline \multirow{4}{*}{ E-commerce platform website trust } & D1 & $.721(* *)$ \\
\hline & D2 & $.684(* *)$ \\
\hline & D3 & $.699(* *)$ \\
\hline & D4 & $.689(* *)$ \\
\hline
\end{tabular}

(2) Reliability analysis of latent variables

Figure 4 shows that each potential variable's alpha coefficients are above 0.6 , which is an acceptable range. Among them, the effectiveness of the third-party payment instrument, the effectiveness of the communication and interaction mechanism, and the reliability of the transaction subject purchase-intention measure are relatively high.

\section{(3) Validity analysis}

The construct validity is based on whether it is related to the measurement item between the same variables. The correlation coefficient between the scores of individual items and the total score is used to verify the scale. When the correlation coefficient is significant, the scale has good construct validity. If the correlation coefficient is not significant, it is necessary to remove individual items, with validity retested. When the measurement item's total correlation coefficient is less than 0.5 , the corresponding measurement item can usually be deleted. The work used the SPSS to analyze the construction validity of each potential variable of the questionnaire, as shown in Table 2 (** It is assumed that the hypothesis test (both sides) is significant at a confidence level of 0.01). Figure 5 is obtained from Table 2 . The correlation coefficient between the potential variables and the sum of all the measurable variables is significant. There is no item with low validity in the scale, so the questionnaire's validity satisfies empirical analysis requirements.

(4) Analysis of the choice of credit mechanism in e-commerce enterprises

In Figure 6, the cost of operating a fully decentralized credit system in the e-commerce market increases as transactions increase; the operating costs of third-party credit intermediaries increase as transactions increase in a small range. E-commerce companies located on the left side of the two curves 
Figure 5. Relevance validity analysis of individual and total variables of each potential variable

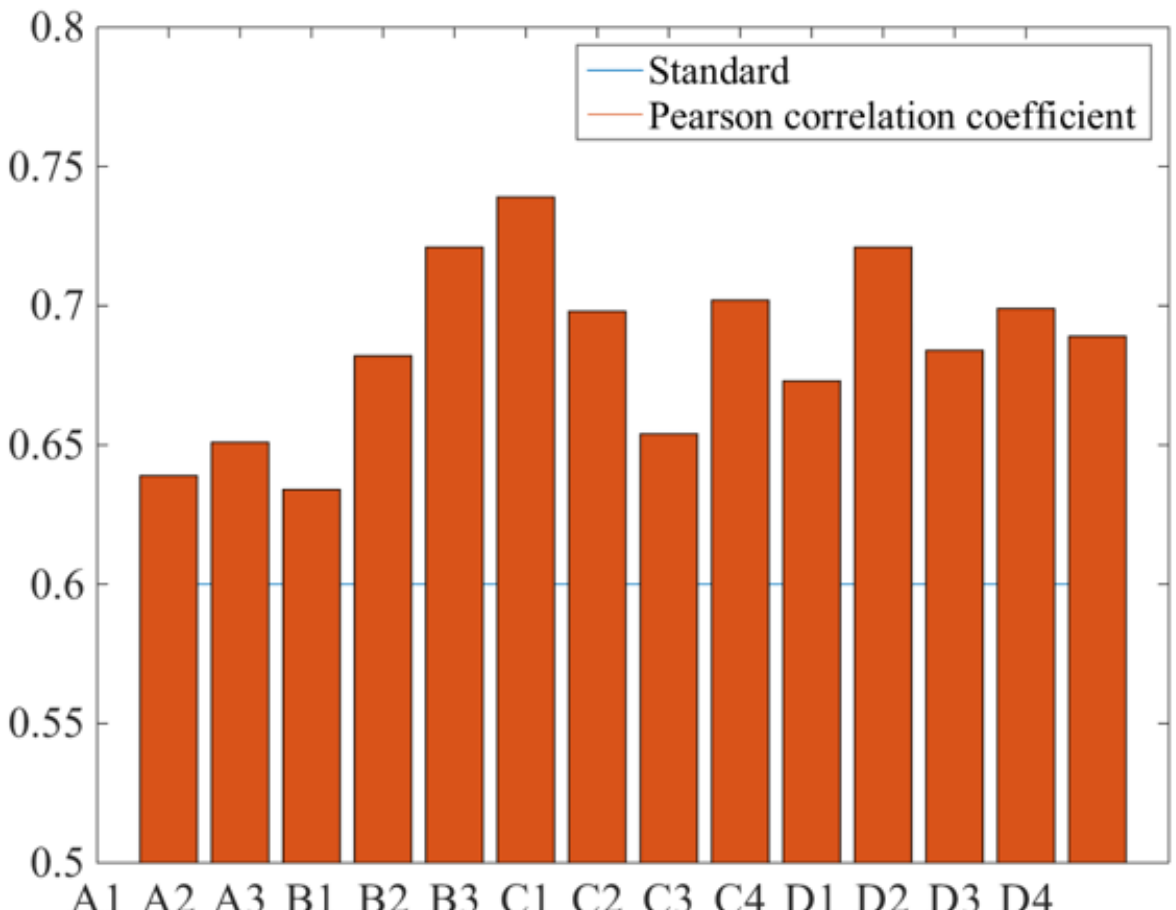

Figure 6. Cost comparison of e-commerce companies under different credit mechanisms

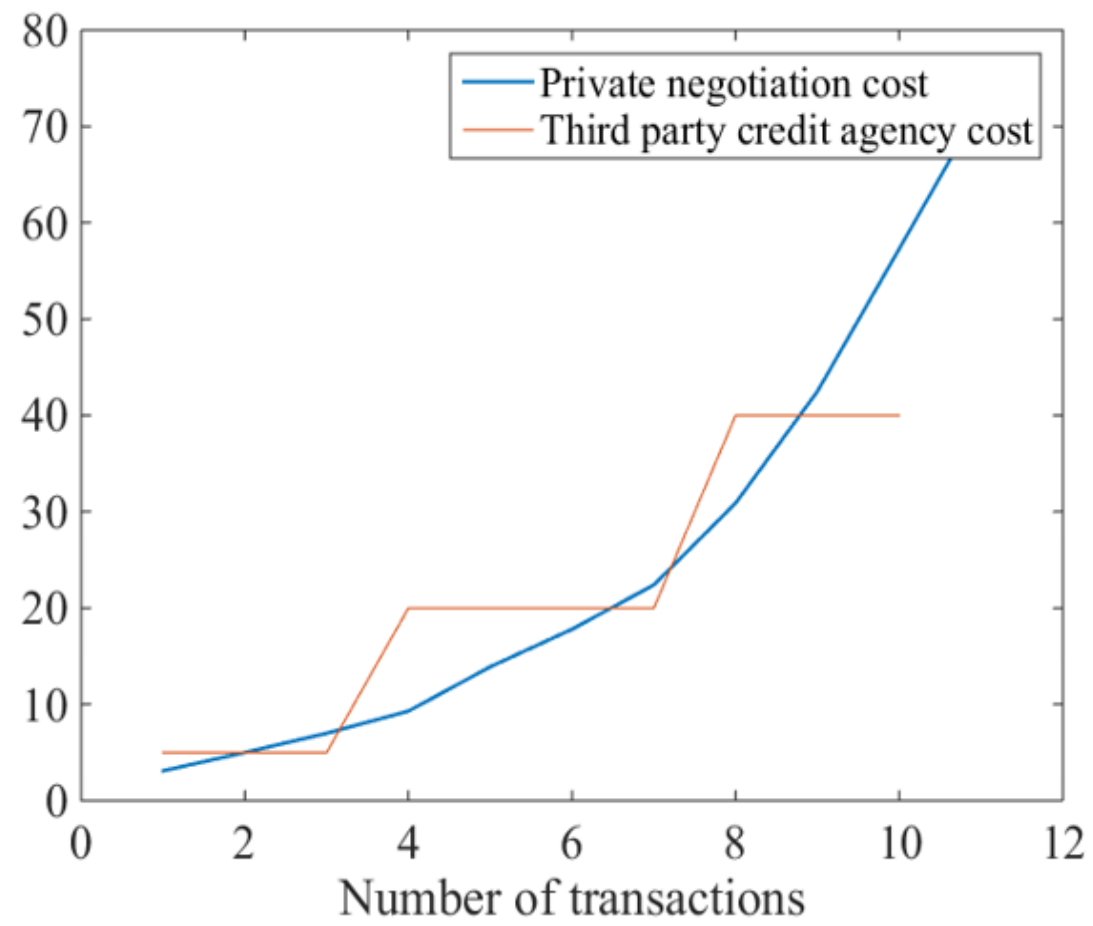


with the transaction volume of $\mathrm{T}^{*}$ do not introduce third-party credit intermediaries, and e-commerce companies with transaction volumes exceeding $\mathrm{T}^{*}$ introduce third-party credit intermediaries. For e-commerce companies whose transaction volume is exactly equal to $\mathrm{T}^{*}$, there is no apparent tendency to choose two credit mechanisms. The choice of credit mechanism for e-commerce companies is related to the net income they have achieved under each credit mechanism. E-commerce companies that are smaller and have fewer transactions are unwilling to become third-party credit institutions on the Internet. Such companies are more comfortable and unwilling to pay for such credit services. If encountering a credit fraud complaint, they negotiate with the consumer in private. On the other hand, large e-commerce companies take the lead in supporting the introduction of third-party credit intermediaries because they benefit more.

\section{CONCLUSIONS}

In the context of e-commerce, corporate marketing strategies present new characteristics, and corporate marketing models, marketing channel expansion, online promotions, and e-commerce-based customer relationship management must be innovated. In e-commerce, companies will devote themselves to customized marketing that meets individual needs based on homogeneity. That is to say, only under the conditions of e-commerce can "one-to-one" marketing be realized. The product life cycle is greatly shortened. More and more product categories and more and more customers' options lead to more and more transaction modes. Information technology has also established the dominant position of information flow in marketing channels, and objectively puts forward requirements for transforming enterprises' traditional channels. The new channels in the e-commerce environment should be traditional channels that have been transformed and integrated according to e-commerce requirements. E-commerce, with its characteristics of no geographic spatial boundaries, low cost of customer communication, and powerful customer relationship processing functions, also supports enterprise customer relationship management.

The work was based on the e-commerce environment to develop security factors in the process of commodity trading in good social relations: taking credit mechanism as the research object, using game theory, information asymmetry, and other related theories as the theoretical basis, running through the e-commerce market economy characteristics and users. The credit behavior of the transaction fully condensed the existing "micro" and "macro" research foundations, established the model and mechanism of the "middle" level e-commerce environmental credit mechanism, and conducted game theory analysis and empirical research. Combined with the relevant mechanism of action and existing research results, we discussed the credit mechanism's construction strategy in China's e-commerce environment. Strong support was provided for the construction and development of the methods to maintain social relations in the process and China's e-commerce environment commodity trading.

First, by systematically analyzing the credit problem and its related economic theory, the credit mechanism should be regarded as an economic system. From the development of the credit mechanism, the credit mechanism initially relied on specific informal credit rules. With the rapid development of the market economy, the credit relationship increasingly depended on protecting the formal credit system represented by laws and regulations. Also, the credit mechanism's final formation was the result of a group choice, so any country or nation had its uniqueness in its credit mechanism's design and choice. Therefore, to build a system of social relations maintenance in e-commerce transactions in China must be based on China's national conditions. Secondly, through the construction of the basic model of the e-commerce market, This paper started from the root of the influencing factors of the credit problem of maintaining social relations in e-commerce environmental commodity trading and used the game theory method to solve the problem of information asymmetry and government market regulation. The related subjects of credit mechanism and their relationship in the e-commerce environment were deeply analyzed. 
Through theoretical research, game theory analysis, and empirical research, the work analyzed the credit mechanism and construction strategy of maintaining social relations in e-commerce environmental commodity trading and supplemented the research on maintaining social relations in China's e-commerce to a certain extent. During writing the thesis, we find that the following aspects are worthy of further research: First, at the macro-policy level, the e-commerce credit control policy needs further research. Although the work used absolutism theory to conclude that in the e-commerce market, the credit mechanism based on the cooperation between the government and third-party credit intermediaries can achieve the best social benefits. However, the question that arises is what policies in e-commerce to maintain social relations are in line with the method for e-commerce to maintain social relations mechanisms? How do the relevant policies for maintaining social relations in e-commerce and environmental commodity transactions in developed countries in Europe and the United States come out and operate effectively? Which specific regulations on e-commerce environmental commodity trading in China have been formulated? What is the effect? How should the next step be improved? Therefore, further research is required.

\section{ACKNOWLEDGMENT}

The work was supported by the 2020 project of the 13th Five-Year Plan for Education Science of Hunan Province: Research on the construction of first-class undergraduate Major of applied E-commerce under the background of "Shuangwan Plan" (Grant No. ND205702); General Funded project of Hunan Social Science Achievement Evaluation Committee, "Research on the Path and Policy of Promoting the Development of E-commerce of Agricultural Products in Hunan province", (XSP17YBZZ066). 


\section{REFERENCES}

Aanen, S. S., Vandic, D., \& Frasincar, F. (2015). Automated product taxonomy mapping in an e-commerce environment. Expert Systems with Applications, 42(3), 1298-1313. doi:10.1016/j.eswa.2014.09.032

Bhadoria, R. S., \& Chaudhari, N. S. (2019). Pragmatic Sensory Data Semantics with Service-Oriented Computing. Journal of Organizational and End User Computing, 31(2), 22-36.

Chen, C., Pan, S., Wang, Z., \& Zhong, R. Y. (2016). Using taxis to collect citywide E-commerce reverse flows: A crowdsourcing solution. International Journal of Production Research, 55(7), 1833-1844. doi:10.1080/002 07543.2016 .1173258

Chen, J. V., Yen, D. C., Pornpriphet, W., \& Widjaja, A. E. (2015). E-commerce web site loyalty: A cross-cultural comparison. Information Systems Frontiers, 17(6), 1283-1299. doi:10.1007/s10796-014-9499-0

5Hua, N. (2016). E-commerce performance in hospitality and tourism. International Journal of Contemporary Hospitality Management, 28(9), 2052-2079. doi:10.1108/IJCHM-05-2015-0247

21. Jin, S., Li, H., \& Yao, L. (2017). Preferences of Chinese consumers for the attributes of fresh produce portfolios in an e-commerce environment. British Food Journal, 119(4), 817-829. doi:10.1108/BFJ-09-20160424

Kabanda, S., \& Brown, I. (2017). Interrogating the effect of environmental factors on e-commerce institutionalization in Tanzania: A test and validation of small and medium enterprise claims. Information Technology for Development, 23(1), 59-85. doi:10.1080/02681102.2016.1263593

1Kaluzhsky, M. (2015). Institutionalization of the payment environment of e-commerce in Russia. Social Science Electronic Publishing, 8(3), 1363-1369.

Kim, H. (2019). Investigating the Mediating Role of Social Networking Service Usage on the Big Five Personality Traits and on the Job Satisfaction of Korean Workers. Journal of Organizational and End User Computing, $31(1), 110-123$.

Kj, L. L., Aresu, A., \& Stephanou, S. (2015). The Commission's E-Commerce Sector Inquiry: Analysis of Legal Issues and Suggested Practical Approach. Social Science Electronic Publishing, 6(7), 465.

Maier, D. S. (2018). Should biodiversity and nature have to earn their keep? What it really means to bring environmental goods into the marketplace. Ambio, 47(4), 477-492. PMID:29243053

Mohanty, B. K., \& Gupta, M. (2015). Product Selection in E-Commerce Under Fuzzy Environment: A MADM Game-Theoretic Model. International Game Theory Review, 17(01), 1540008. doi:10.1142/S0219198915400083

Mohbey, K. K., \& Thakur, G. S. (2015). Interesting User Behaviour Prediction in Mobile E-commerce Environment using Constraints. IETE Technical Review, 32(1), 16-28. doi:10.1080/02564602.2014.968224

Song, S. M., Kim, E., Tang, R., \& Bosselman, R. (2015). Exploring the Determinants of e-Commerce by Integrating a Technology-Organization-Environment Framework and an Expectation-Confirmation Model. Tourism Analysis, 20(6), 689-696. doi:10.3727/108354215X14464845878156

Tamini, L. D., \& Sorgho, Z. (2018). Trade in Environmental Goods: Evidences from an Analysis Using Elasticities of Trade Costs. Environmental and Resource Economics, 70(1), 53-75. doi:10.1007/s10640-017-0110-2

Taylor, L. O. (2016). Incentive Compatible Referenda and the Valuation of Environmental Goods. Agricultural and Resource Economics Review, 27(2), 132-139. doi:10.1017/S1068280500006456

Uzoka, F. M. E., Seleka, G. G., \& Khengere, J. (2017). E-commerce adoption in developing countries: A case analysis of environmental and organizational inhibitors. International Journal of Information Systems and Change Management, 2(3), 232-260. doi:10.1504/IJISCM.2007.015598

Wei, C. L., \& Ho, C. T. (2019). Exploring Signaling Roles of Service Providers' Reputation and Competence in Influencing Perceptions of Service Quality and Outsourcing Intentions. Journal of Organizational and End User Computing, 31(1), 86-109.

Yunita, I. R., \& Gunawan, W. (2018). The influence of social media to loyalty in an e-commerce. IOP Conference Series. Materials Science and Engineering, 420(1), 012102. 
Zhang, H., Wang, Y., Zhang, X., \& Lim, E.-P. (2015). ReputationPro: The Efficient Approaches to Contextual Transaction Trust Computation in E-Commerce Environments. ACM Transactions on the Web, 7(9), 1331-1351. doi: $10.1145 / 2697390$

Zhang, L. X. Z., Mouritsen, M., \& Miller, J. R. (2019). Role of Perceived Value in Acceptance of "Bring Your Own Device" Policy. Journal of Organizational and End User Computing, 31(2), 65-82.

Zhou, S., Sun, B., \& Ma, W. (2017). The pricing strategy for Fuji apple in Shaanxi of Chain under the e-commerce environment. Kybernetes, 47(7), 208-221. 